\title{
A Short Model Excitation of An Asymmetric Force Free Superconducting Transmission Line Magnet
}

\author{
M. Wake, H. Sato, R. Carcagno, W. Foster, S. Hays, V. Kashikhin, A. Oleck, H. Piekarz, and R. Rabehl
}

\begin{abstract}
A short model of asymmetric force free magnet with single beam aperture was tested at Fermilab together with the excitation test of VLHC transmission line magnet. The design concept of asymmetric force free superconducting magnet was verified by the test. The testing reached up to $104 \mathrm{kA}$ current and no indication of force imbalance was observed. Since the model magnet length was only $10 \mathrm{~cm}$, A $0.75 \mathrm{~m}$ model was constructed and tested at KEK with low current to ensure the validity of the design. The cool down and the excitation at KEK were also successful finding very small thermal contraction of the conductor and reasonable field homogeneity.
\end{abstract}

Index Terms-VLHC, transmission line magnet, asymmetric, low field superconducting magnet, force free design.

\section{INTRODUCTION}

$A$ prototype of a superconducting transmission line magnet $\mathbf{A}_{\text {system proposed for an injector accelerator in a staged }}$ VLHC p-p collider [1] has been built and successfully tested at Fermilab. A $1.5 \mathrm{~m}$ long, twin-aperture, combined function gradient dipole magnet of $2 \mathrm{~T}$ field is excited by a single-turn $100 \mathrm{kA}$ superconducting transmission line [2]. The force free design of this type of magnet has a great advantage in the cryogenic efficiency and simplicity of the structure making construction of very high-energy accelerator, such as VLHC, realistic. However, the force free feature of this design is based on the symmetry. Therefore, the application of this technology is limited to colliders with twin apertures. The modification of the design to generalize the idea into a general purpose single aperture $\mathrm{C}$ type magnet was proposed by the introduction of a dummy gap filled with stainless steal [3], [4]. The test of VLHC magnet was carried out with very small section of such asymmetric magnet. The cross section of such magnet is shown in Fig. 1. The short model shares the same size of transmission line and has the same $2 \mathrm{~cm}$ vertical gap. The length of the magnet is $10 \mathrm{~cm}$. Since the iron permeability changes with magnetic field, force free design of asymmetric magnet requires the balancing of iron saturation. The force balance can not be perfect at every excitation level but the electro-magnetic force can be designed to the level of practically no-force. It is

Manuscript received September 20, 2005.

M. Wake and H. Sato are with High Energy Accelerator Research Organization, 1-1 Oho, Tsukuba, Ibaraki 305-0801, Japan. (e-mail: wake@ post.kek.jp). R. Carcagno, W. Foster, S. Hays, V. Kashikhin, A. Oleck, H. Piekarz, and R. Rabehl are with Fermi National Accelerator Laboratory, Batavia Illinois, 60510 USA obvious that the $10 \mathrm{~cm}$ magnet length is too short to see the full feature of the design in the excitation test. A $0.75 \mathrm{~m}$ model was also constructed and tested at KEK with low current.

\section{MAgnetic Design AND CONDUCTOR}

The geometry of the asymmetric no-force magnet shares the transmission line of the VLHC magnet with $80 \mathrm{~mm}$ diameter. The pole gap is also the same $2 \mathrm{~cm}$. The magnetic design of the iron yoke was made by the iteration of numerical calculation. The non-linear permeability of the iron is essential to be included in the design. The saturation of the iron can be adjusted by the width and height of the dummy gap and holes in the edge of the gap as shown in Fig. 1. Fig. 2 shows the force balancing result calculated by ANSYS. The force balance is almost perfectly achieved up to $1.6 \mathrm{~T}$. However, it very rapidly becomes unbalanced exceeding $1.6 \mathrm{~T}$. The conductor to be used for this type of magnet has to, of course, carry a very large current. The current of $100 \mathrm{kA}$ is not very difficult for multi-strand cable [5]. Important feature of the cable is to reduce the thermal contraction. Unlike usual superconducting magnet with many turns of thin conductors, this type of magnet does not allow the conductor thermal contraction without deforming the entire magnet system. The solution for this problem is to use braided cable hold in between invar pipes as shown in Fig. 3. Expansion of inner pipe and draw down of outer pipe were found to hold the superconducting braid tight. The thermal shrinkage of the cable can be controlled by this structure of the cable. The measured thermal shrinkage of the

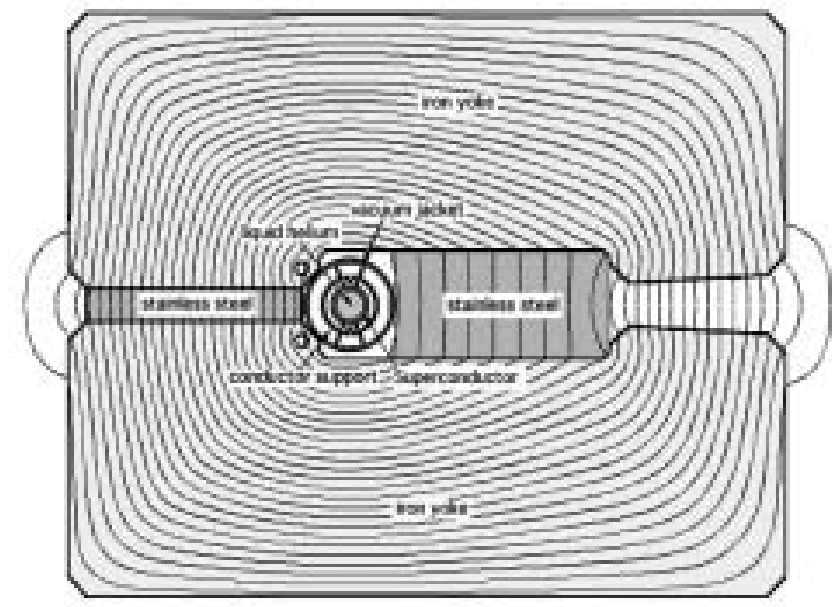

Fig. 1. Structure of asymmetric transmission line magnet. The shape of the magnet is almost the same as a general purpose C-type magnet. The dummy magnetic gap made by non-magnetic material produces a counter force on the conductor to cancel the electro-magnetic force. The real test magnet was built without gradient to check the field. 


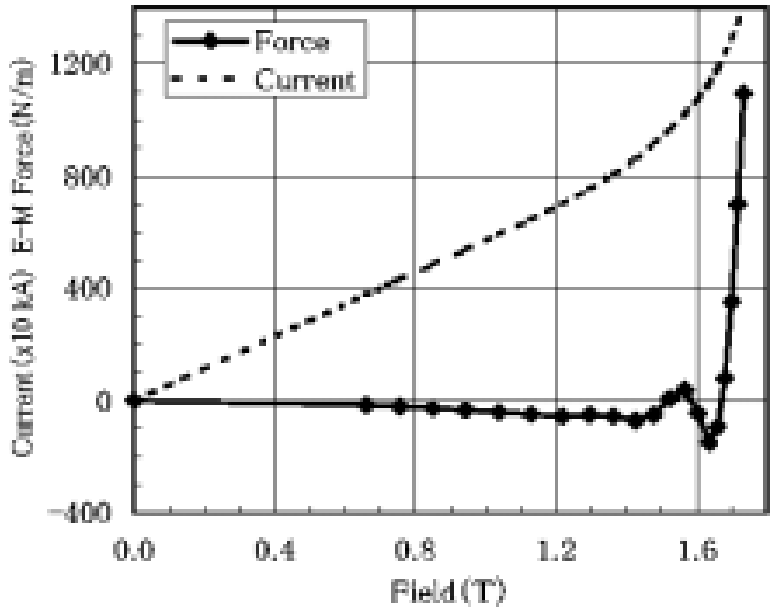

Fig. 2. The force balance and excitation. Force balance is disturbed by the saturation of iron. By the geometrical arrangement of iron, electro-magnetic force can be kept very small until the iron saturation become severe.

cable was $0.05 \%$ from room temperature to $77 \mathrm{~K}$. The cable itself works as the transfer line of the liquid helium.

\section{EXPERIMENTAL SET UP}

The current loop to test the VLHC transmission line magnet was built at MS6 building of Fermilab. The current was supplied through a pair of gas cooled current leads [6]. Each current lead consists of 202 pieces of copper rods with diameter of $6.35 \mathrm{~mm}$ arranged horizontally. The effective length of the current lead is $1650 \mathrm{~mm}$. The copper rods are soft-soldered at both warm and cold ends. The liquid helium gas flow, guided by multiple baffles along the lead length, allows for relatively high heat transfer coefficient. The power supply for the operation of the magnet is a $1.5 \mathrm{~V} 100,000 \mathrm{~A}$ DC switcher power supply [7]. This power supply was used during testing as both the ramping supply and holding supply. The supply consists of a bulk power source, which is filtered and regulated at $400 \mathrm{~V}$ and $240 \mathrm{~kW}$, and 10 switcher cells of 1.5 volt $10,000 \mathrm{~A}$. These forward converter cells are connected in parallel at the input to the power lead. Each converter has a 7:1 turns ratio with the primaries of each of the 112 converters connected in series and the bridge outputs all connected in parallel. During these tests each cell was operated as a constant power device without load current or field feedback. In order to measure the magnetic field harmonics in such a small magnet

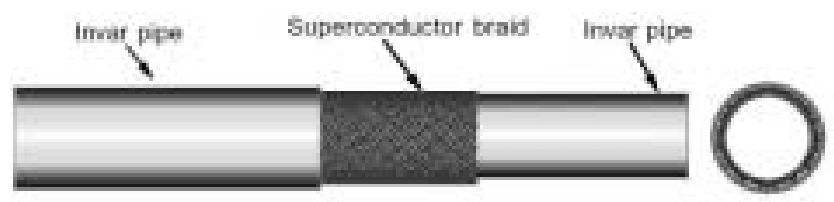

Fig. 3. Cable structure with reduced thermal contraction. 4 x 64 strands of $0.7 \mathrm{~mm}^{\phi} \mathrm{Nb}-\mathrm{Ti}$. Conductor was braided on an invar pipe and covered by another invar pipe. Drawing down and up on a draw bench makes contact between invar pipe and superconductor tight.

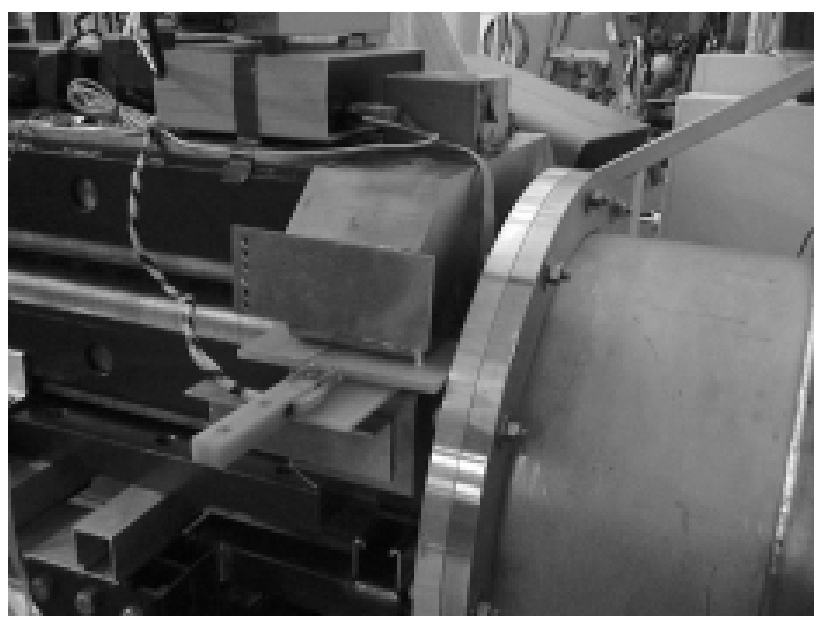

Fig. 4. Asymmetric short model in the testing set up. $10 \mathrm{~cm}$ thick model was mounted at the end of VLHC magnet yoke.

aperture, a specialized rotating-coil probe with $15 \mathrm{~mm}$ diameter and $0.69 \mathrm{~m}$ length tangential coils was fabricated [8]. Another way of field measurement was planed using hall probe array. The asymmetric force free magnet with length $10 \mathrm{~cm}$ was installed at the end of the VLHC magnet as shown in Fig. 4. The current loop is limited in size in this set-up and the length $10 \mathrm{~cm}$ was the maximum available space for the asymmetric magnet.

Liquid helium was supplied from two bottles to cool down the transmission line: one for current leads and the other for the current loop. The cooling was smooth down to $20 \mathrm{~K}$ and humbled because of remaining hot spots in the system. In a liquid helium pipeline with not small aperture, convection makes the helium flow very much complicated. We had to struggle to obtain a good cooling condition of the system. After several attempt of changing helium flows, we reached cooling level sufficient to test the magnets. The power supply was operated gradually to higher current. Since there is no dummy load with inductance available for $100 \mathrm{kA}$, the adjustment of the power supply had to be done at the same time of excitation test. The LABVIEW software was used to monitor the temperature and to record the voltages and current during the

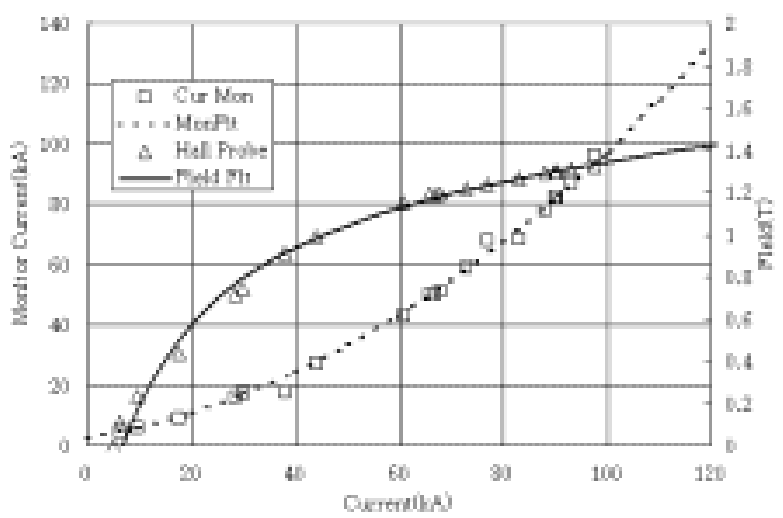

Fig. 5. Excitation of the model magnet. The control current is not necessary the magnet current. The magnet current was estimated by the sum of the each power cell current. The magnetic field was monitored by a hall probe. Inaccuracy at low field was inevitable. 
excitation and quenches. Since there is no $100 \mathrm{kA}$ current transducer, total current had to be determined by adding the output of each current cells. The detail of the experimental set-up is described elsewhere [2].

\section{Excitation Results}

The transmission line loop was ramped in trapezoidal wave shape with a 10 second flat top. The excitation was repeated gradually increasing the maximum current. This was necessary for the adjustment of the power supply. Fig. 5 depicts the excitations made during the test. The nominal current in the horizontal axis is the measure of the power input to the power supply modules and does not necessary mean the actual current. The magnet current was measured by the sum of the each power supply cell after the excitation. The test was interrupted many times to change the liquid helium bottles. We used $12000 \mathrm{~L}$ of helium in total. The helium consumption at the leads was not as much as we anticipated [6] because the current was not large most of the time during the test. The excitation reached to the design current of $75 \mathrm{kA}$ with no quench. The quench of the magnet occurred at the end of the flat top during the ramp with maximum current 104 kA. Fig. 6 shows the current and voltages during the quench in two different time scales. The voltage was built up at first in the VLHC magnet section and propagated into the return path. The asymmetric force-free magnet was in the return end side of the VLHC magnet Therefore, the force imbalance in the asymmetric magnet is not the cause of the quench.

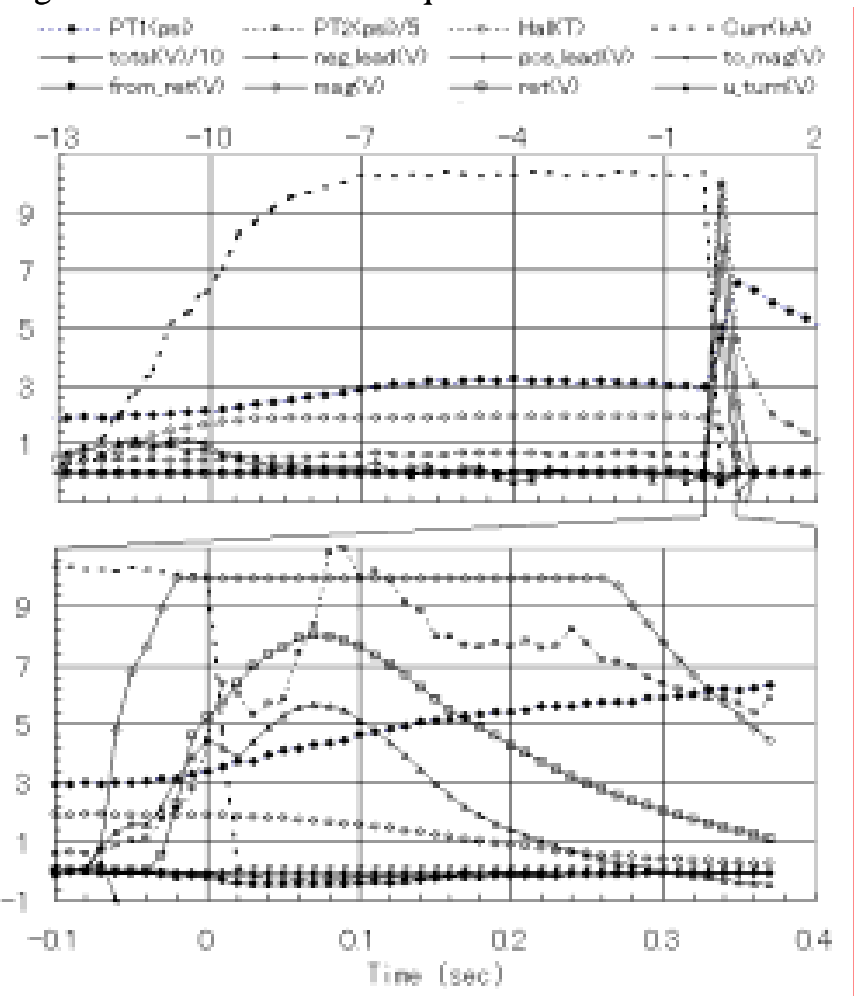

Fig. 6. The voltages and current observed during the $104 \mathrm{kA}$ quench. Bottom is the enlarged plot of the quench development. Mag (V) is the first to show voltage and propagates in to U-turn (V). The starting of the quench is determined to be at the VLHC magnet section. The asymmetric model is at the entrance of U-turn.

This quench had started at the hot spot in the magnet section. Since the quench current was over the design value, the excitation test achieved the verification of the VLHC design. Since there is no way of extracting the stored energy, the quench had to be violent. The pressure in the helium volumes was at the edge of the ceramic joint strength. The field measurement in the VLHC magnet was made both by a rotating coil and hall probe array. The field in the asymmetric model magnet was measured by a hall probe array [9]. The field quality measurement results with a hall probe array in the asymmetric model are shown in Fig. 7. The calibration error and measurement noise were too large to determine the high-order harmonic components. However, the sextupole and quadrupole components were obtainable by curve fitting. The results were found reasonably small as designed. The magnetic field of this model was expected to saturate at $1.6 \mathrm{~T}$ by the 2-D calculation but saturation was observed at $1.4 \mathrm{~T}$. This difference is due to too short length of the model. Because of the large deviation from design in the saturation field, it is not very clear if the no-force situation was really achieved or not. However, the motion of the yoke due to the unbalanced force was not observed, namely, quenches were not in the position of the asymmetric magnet. The first excitation of the asymmetric no-force magnet was achieved with no indication of fundamental problem.

\section{0.75 METER MODEL}

Since the $10 \mathrm{~cm}$ model is too short to avoid the end effect, a $0.75 \mathrm{~m}$ model in Fig. 8 was built at KEK and tested at low current. A battery operated power supply [10] capable of supplying $2000 \mathrm{~A}$ was used. This magnet has the same cross section as the $10 \mathrm{~cm}$ model tested at Fermilab. The cooling of the transmission line was made by liquid helium flow between two reservoirs in both ends of the transmission line. Such arrangement, with both ends fixed geometry, is possible only for non-contraction transmission line. If there is too much

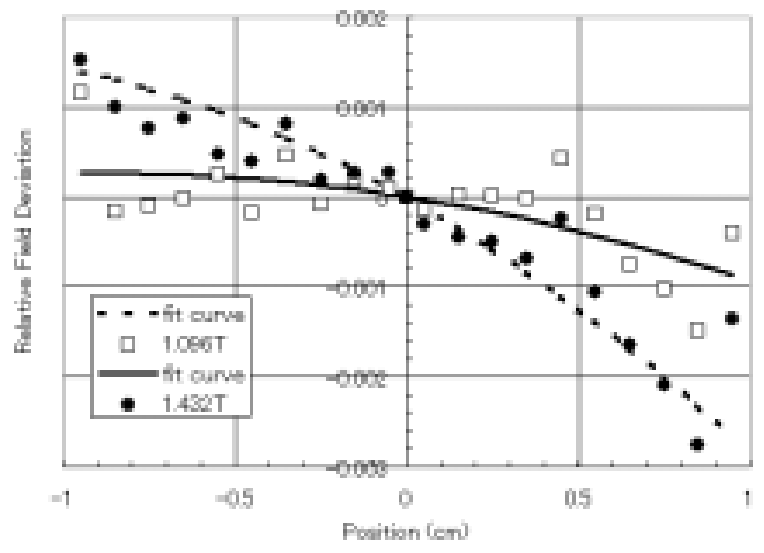

Fig. 7. Hall probe array measurement. An array of 20 hall probes mapped the field. Measured $b_{1}, b_{2}$ for $1.1 \mathrm{~T}$ and $1.4 \mathrm{~T}$ were $-5.9 \times 10^{-4}$, $-3.4 \times 10^{-4},-2.1 \times 10^{-3}$ and $-3.4 \times 10^{-3}$ at $1 \mathrm{~cm}$ respectively. 
contraction in the transmission line, the ceramic insulation of the conductor has to break. Therefore the cooling of the cryostat is an experiment to verify the characteristics of the non-contraction cable. The cooling was successful with no failure in vacuum. Another attempt made in the low current test was the absolute measurement of the field at very low current. An electron spin resonance (ESR) device was used to measure the field to $6^{\text {th }}$ digit. The field measurement result is shown in Fig. 9. The multipole component of the field can be obtained by the fitting to the polynomial:

$$
\begin{aligned}
B(x)=B_{0}\left(1+b_{1} x+\right. & b_{2} x^{2}+b_{3} x^{3} \\
& \left.+b_{4} x^{4}+b_{5} x^{5}+b_{6} x^{6}\right)
\end{aligned}
$$

The quadrupole $\left(b_{1}\right)$, sextupole $\left(b_{2}\right)$, octapole $\left(b_{3}\right)$, decapole $\left(b_{4}\right)$, dodecapole $\left(b_{6}\right)$ components were $-1.4 \times 10^{-4}$, $2.27 \times 10^{-3}, 3.17 \times 10^{-4}, 8.21 \times 10^{-4}$, and $-1.7 \times 10^{-4}$ at $1 \mathrm{~cm}$ respectively. ESR measurement is effective giving enough digits to determine high order multipole components. The field homogeneity at low field is usually a problem for superconducting magnets but the $0.5 \%$ flat field region was 3 $\mathrm{cm}$ even at very low field in this magnet as shown in Fig. 9. The transmission line magnet could be used with very low injection field. The $0.75 \mathrm{~m}$ model was made not with laminated iron but with a machined solid iron. The geometrical accuracy of $10^{-4}$ for the pole gap of $2 \mathrm{~cm}$ is $2 \mu \mathrm{m}$ and this is very tight. A gap polishing after the assembly of the iron may be necessary to truly ensure the magnetic field accuracy.

\section{CONCLUSION}

The first excitation of asymmetric no-force magnet was successfully achieved together with the test of the VLHC transmission line magnet. There was no symptom of force imbalance within the observation in the testing. The test of $0.75 \mathrm{~m}$ model at low field verified the field homogeneity of the iron-dominated magnet. The injection field of accelerators using superferric magnet could be very low. The cool-down of $0.75 \mathrm{~m}$ model also gave confidence for the non-contraction

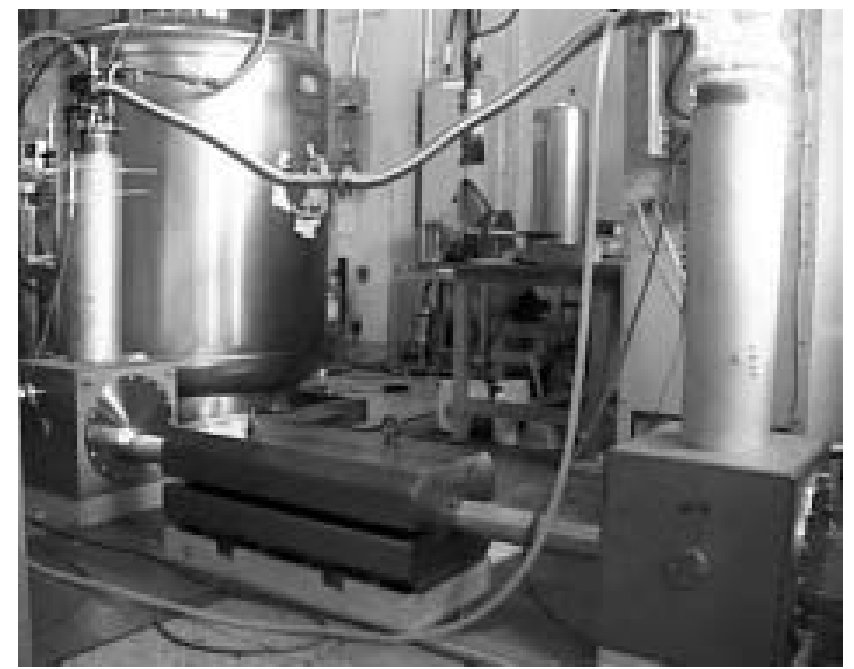

Fig. 8. The $1 \mathrm{~m}$ asymmetric model built at KEK. The cooling of the transmission line was made by the liquid flow between supply bottle and outlet bottle.

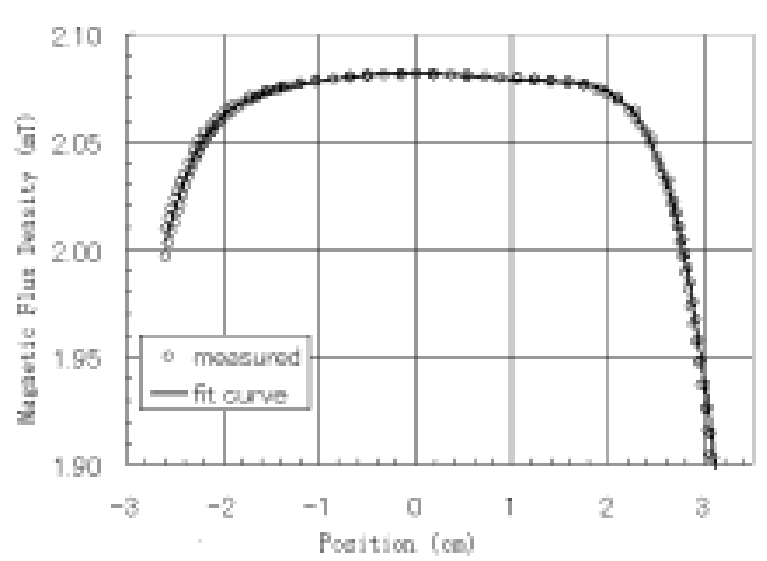

Fig. 9 Magnetic flux density profile at low current. The ESR field measurement results at current 66.0 A. EFM-30AX Field meter (Echo Electric Co.) converts the resonant frequency of DPPH into the magnetic field. Probe moving stage is controlled in $20 \mu \mathrm{m}$ step and supposed to have better accuracy.

cable. The concept of asymmetric no-force magnet was given some confidence by the experiments. The wide application of this new superconducting magnet is expected in the future.

\section{ACKNOWLEDGMENT}

The authors are grateful to Mr. Y. Kobayashi of KEK Mechanical Engineering Center for his engineering effort in the cryostat construction.

\section{REFERENCES}

[1] "Design study for a staged very large hadron collider", Fermilab-TM-2149, 2001.

[2] H. Piekarz, W. Foster, S. Hays, Y. Huang, V. Kashikhin, A. Oleck, R. Rabehl, P. Schlabach, G. Velev; M. Wake, "Design, fabrication and test of a 2 Tesla superconducting transmission line magnet system", IEEE Trans. Supercond., submitted for publication, 2005.

[3] M.Wake and R.Yamada, "New types of super-conducting magnet designs for high energy collider", Proc. MT-15 pp. 47-50, Science Press, Beijing, 1998.

[4] M.Wake, H.Sato, K.Takayama, H.Piekarz, R.Yamada, "Force-free design of beam line magnet", proc. proc.2003 Particle Accelerator Conf. p. 1981, 2003.

[5] E.Barzi, G.W.Foster, E.Malamud, P.O.Mazur, H.Piekarz, M.Wake, K.Hayashi, M.Koganeya, "Nb3Al prototype conductor for the transmission line magnet", proc.1999 Particle Accelerator Conf. pp. 3330-3332, 1999.

[6] Y. Huang, W. Folster, S.W. Kim, P. Mazur, A. Oleck, H. Piekarz, R. Rabehl, and M. Wake, "The $100 \mathrm{kA}$ current leads for a superconducting transmission line magnet", IEEE Trans. Supercond., submitted for publication, 2005.

[7] L. Hays, B. Claypool, W. Foster, "The 100 kA DC Power Supply for a Staged Hadron Collider Superferric Magnet", IEEE Trans. Supercond., submitted for publication, 2005.

[8] G.V. Velev, W. Foster, V. Kashikhin, P. Mazur, A. Oleck, P. Schlabach, C. Sylvester, and M. Wake, "Field quality measurements of a 2-Tesla superconducting transmission line magnet", IEEE Trans. Supercond., submitted for publication, 2005.

[9] V. Kashikhin, R. Carcagno, W. Folster, R. Nehring, H. Piekarz, P. Schlabach, "Test results of a 2 Tesla superconducting transmission line magnet obtained with 102 sensor array of hall station", IEEE Trans. Supercond., submitted for publication, 2005.

[10] M.Wake, H.Sato, K.Suda, "A Battery-Powered High-Current Power Supply for superconductors (in Japanese)" Teion-kogaku, 37 pp. 783-786, 2002. 